\title{
LUMINOSITY FUNCTION OF OLD GLOBULAR CLUSTERS IN THE SMC
}

\author{
M. Kontizas and E. Kontizas \\ University of Athens, Observatory of Athens
}

\section{INTRODUCTION}

Luminosity Functions (LFs) provide useful information on the stellar content of a star cluster. One of the problems of studying the colour-magnitude $(c-m)$ diagrams in remote clusters is the contamination of cluster members with field stars. If all the stars of a cluster are measured in concentric rings one can reach the field and compare the c-m diagram of the field with that of the central areas. For the SMC where the field and cluster areas seem to have similar c-m diagrams, this method is not always useful and then the LF becomes an important tool of overcoming this obstacle.

LFs of 10 SMC star clusters have been derived using photographic plates taken with the $1.2 \mathrm{~m} U . K$. Schmidt telescope. The large field schmidt offers the advandage of having homogeneous photographic material for both the clusters and their adjoining fields.

The photometric values were found by means of an irisphotometer and the accuracy of the measurements was discussed by Kontizas (1980). Star counts in the central areas of the clusters and their adjoining fields have provided the necessary normalising factors which were used to subs-

tract the LF of the field from the corresponding LF of the central area.

The LF of NGC152 derived from the Schmidt plates was found to be in good agreement with that found from AAT plates.

DISCUSSION

The study of the LEs of 10 old globular clusters, loca- 
ted in the western and the northern areas has shown the following :

(i) the bright red stars belong to the clusters and if there are red field stars they are certainly much fainter.

(ii) in most cases comparison, with the available theoretical models of globular clusters, has failed to match mainly because the subgiant part is very steep (may be due to helium content; Kontizas and Kontizas, 1982 ) and the horizontal branch (HB) is very populous and has only the red part of the RR Lyrae strip. This HB structure would mean high metallicity but we know that this is not the case for the SMC. So it can be assumed that the populous HB could be produced by a clump of intermediate age stars (more massive than those found in old globulars) as is the case of old open clusters of our Galaxy. In our sample the clusters I11 and NGC152 have this characteristic particularly prominent. From the c-m diagram of these ten clusters, it was found that L11 and NGC152 are amongst the youngest (Kontizas, 1976; Hodge, 1982 ). So the structure of the LF where the HB is expected could be an age indication and therefore the old clusters of the SMC seem to be younger than the corresponding old globulars of our Galaxy and contain more massive star members.

(iii) The field LF seems to resemble the LFs of the oldest SMC clusters of our sample. This field is purely SMC halo stars since the stars of our Galaxy give almost negligeable contribution at this part of the sky ( Brück and Marsoglu, 1978 ).

\section{ACKNOWLEDGMENTS}

The authors are very much obliged to the $1.2 \mathrm{~m}$. U.K. Schmidt telescope Unit for providing the necessary phtographic material.

\section{REFERENCES}

Brück, M. T. and Marsoglu, A., : 1978, Astron. and Astrophys. Hodge $\frac{68}{\mathrm{P}}, 193$ : 1982 , IAU Colloquium No 68, pp. 205

Kontizas, M. : 1976 , PhD Thesis, Edinburgh University.

Kontizas, M. : 1980 , Astron. and Astrophys. Suppl. Ser., $40,151$.

Kontizas, M., and Kontizas, E. : 1982, Astron. and Astrophys 108,344 . 


\section{DISCUSSION}

V.M. BLANCO: In one of the viewgraphs you showed, a magnitude limit was indicated for the available information about the clusters luminosity functions. I would like to remark that in the forthcoming Magellanic Clouds observing season many SMC and LMC globular clusters will be obser ved at CTIO with a CCD camera on the 4-meter telescope. From work done during the past season on a few clusters, for example Kron 3, we expect the observations to extend well past the mean sequence turnoffs of the clusters. Thus, within a year we should have greatly improved information about the luminosity functions of the clusters. Also, you remarked that some clusters showed carbon stars. Generally those are the Searle et al. class IV and V and perhaps VI clusters. It is interesting that Perssons and Frogel find that in such clusters the carbon stars contribu te about $50 \%$ of the total bolometric luminosity. By comparison, in the field (actually a sample field in the LMC) the carbon stars contribute only about 3\% of the total luminosity. Finally, I would like to congratu late you for your beautiful work.

M. KONTIZAS: Thank you very much for your comments. I'll be glad to see the new material. 